\title{
Immunogenicity and immunoreactivity of Tp0821 recombinant protein from Treponema pallidum
}

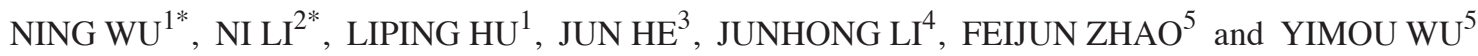 \\ ${ }^{1}$ Department of Clinical Laboratory, The First People's Hospital of Hengyang, Hengyang; ${ }^{2}$ Emergency Department, Second \\ Affiliated Hospital, University of South China, Hengyang; ${ }^{3}$ Department of Clinical Laboratory, Nanhua Affiliated Hospital, \\ University of South China, Hengyang, Hunan $421001 ;{ }^{4}$ Changzhou Center for Disease Control and Prevention, Changzhou, \\ Jiangsu 213022; ${ }^{5}$ Pathogenic Biology Institute, University of South China, Hengyang, Hunan 421001, P.R. China
}

Received March 20, 2016; Accepted February 13, 2017

DOI: $10.3892 / \mathrm{mmr} .2017 .6675$

\begin{abstract}
Treponema pallidum (Tp) is responsible for invading reproductive organs and the skin in early stages, and involves almost all organs/systems at advanced stages. In the present study, screening of the dominant epitope fragment of the Tp outer membrane protein, Tp0821, was performed and the prokaryotic expression vector $\mathrm{pQE} 32 / \mathrm{Tp} 0821$ was constructed. The denaturation and dialysis of rTp0821 were achieved through ultrasound, inclusion body washing and dissolution. Experiments in purified rTp0821-immune New Zealand rabbits indicated that the recombinant proteins were of high immunogenicity, and the irritation led a marked humoral immune response in the New Zealand rabbits. Western blot analysis showed that the purified recombinant proteins reacted with the Tp-positive infected serum, confirming the high level of immunoreactivity. The delayed type hypersensitivity of rTp0821 recombinant proteins was positive, indicating that rTp0821 induced a specific cell immune response and was selected as a Tp vaccine candidate protein. The findings of the present study provided novel evidence, which provided information for further investigations on the pathogenic mechanism of $\mathrm{Tp}$ and the development of diagnostic reagents.
\end{abstract}

\section{Introduction}

Treponema pallidum (Tp) is a pathogen, which causes syphilis, a health-threatening sexually transmitted disease. During the long disease course, syphilis invades reproductive organs and the skin at the early stage, and involves almost all organs/systems at the advanced stage $(1,2)$. The only infective

Correspondence to: Dr Ning Wu, Department of Clinical Laboratory, The First People's Hospital of Hengyang, 36 Hubei Road, Zhuhui, Hengyang, Hunan 421001, P.R. China

E-mail: rencaoer@163.com; 11871541@qq.com

*Contributed equally

Key words: Treponema pallidum, immunogenicity, immunoreactivity source of syphilis is humans, and the disease can be divided into congenital syphilis or acquired syphilis depending on the mode of infection $(3,4)$. Although a suitable drug for syphilis, penicillin, has been identified, the incidence of syphilis has increased in previous years (5). Thus, investigating the pathogenic mechanism of Tp and its diagnostic methods is important for the prevention and control of syphilis.

At present, common laboratory detection methods include direct pathogen detection, serological assessment and polymerase chain reaction (PCR) analysis (6). In particular, serological assessment is commonly used in the clinical screening and diagnosis of syphilis, and primarily includes non-Tp and Tp antigen serum tests (7). The non-Tp antigen serum assessment utilizes non-specific lipoids as antigens and detects blood reagin as a syphilitic marker (8). The Tp antigen serum assessment utilizes Tp or its bacterial components as the antigens, and enzyme-linked immunosorbent assay (ELISA) based on recombinant antigens has gradually been used for confirmation of Tp infection (9).

With the rapid development of gene engineering technology and Tp whole-gene sequencing, the use of syphilis antibody serological methods with the single or combined use of four recombinant proteins (TpN47, TpN17, TpN15 and TmpA) as the envelope antigen has shown high sensitivity and specificity (10-12). However, it has been shown that these recombinant antigens are usually completely natural $\mathrm{Tp}$ antigens, which have cross-reacted with other Spirochaetae (13). These methods cannot detect syphilis at all stages, and their specificity and sensitivity require further improvement (6). There is no consensus regarding which Tp protein antigen has a higher specificity and sensitivity in the serologic diagnosis of syphilis (14). Thus, additional proteins require identification to achieve rapid syphilis diagnosis.

As reported previously, Tp0821 has high antigenicity and its crystal structure is similar to that of cytoplasmic ligand-binding proteins $(15,16)$. Tp0821 is also homologous with the methionine transport protein family and may be involved in the Tp methionine transport system (16). In the present study, using bioinformatics software for analysis, the dominant epitope region of Tp0821 was cloned and purified rTp0821 was expressed. The rTp0821 was used to immunize New Zealand rabbits, and polyclonal antibody titers were 
detected to assess immunogenicity. Using serum from syphilitic patients as a primary antibody, the immunoreactivity of recombinant proteins was detected using western blot analysis and indirect ELISA. The role of rTp0821 in syphilis serologic diagnosis was further evaluated from the perspectives of immunoreactivity and immunogenicity, which underlies the pathogenic mechanism of $\mathrm{Tp}$.

\section{Materials and methods}

Materials. An Ni-NTA Fast Start kit was purchased from Qiagen China Co., Ltd. (Shanghai, China). A bicinchoninic acid (BCA) protein assay kit, goat anti-human IgG/horseradish peroxidase (HRP) and goat anti-rabbit IgG/HRP were purchased from Beyotime Institute of Biotechnology (Haimen, China). 6-His-Tag was purchased from Bioss (Beijing, China). Detoxi-Gel $^{\mathrm{TM}}$ Endotoxin Removing Gel was purchased from Thermo Scientific, Inc. (Waltham, MA, USA). A Limulus Amebocyte Lysate kit was purchased from Limulus Reagent Factory (Xiamen, China). ECL system was purchased from GE Healthcare Bio-Sciences (Pittsburg, PA, USA). The DNA Ladder (1 Kb), BamHI, NotI and T4 ligase were purchased from Takara Biotechnology Co., Ltd. (Dalian, China). The protein marker was purchased from Solarbio (Beijing, China). The Plasmid Miniprep kit and PCR Product Purification kit were purchased from Omega Bio-Tek, Inc. (Norcross, GA, USA). The ELISA kit was purchased from eBioscience, Inc. (Vienna, Austria).

From December 2014 to December 2015, a total of 150 samples of syphilis-negative serum were acquired from the Department of Blood Transfusion, the First Affiliated Hospital of South China University (Hengyang, China). There were 98 males and 52 females, from 18 to 67 years old (mean 45.4 years), the samples of syphilis-negative serum were used as fluorescent treponemal antibody-absorption (FTA-ABS)-negative samples. From December 2014 to December 2015, a total of 150 samples of syphilis-positive serum were collected from patients with confirmed syphilis for use as FTA-ABS-positive samples. There were 87 males and 63 females, from 18 to 64 years old (mean 44.8 years), the samples of syphilis-positive serum were acquired from the Department of Clinical Laboratory, the First Affiliated Hospital of South China University and the Second Affiliated Hospital of South China University. All experiments involving human patients were performed following the provision of informed consent and ethical approval.

Strains, cultivation and experimental animals. The pQE32 vector, Escherichia coli strain JM109, E. coli strain M15 were purchased from Qiagen Co., Ltd. (Shanghai, China). Human acute monocytic leukemia suspension cell line (THP-1) and the Tp Nichols strain were obtained from American Type Culture Collection (Manassas, VA, USA). Male healthy New Zealand rabbits ( $n=40$; age, 6 months; weight) were purchased from the Faculty of Experimental Animals at the University of South China (Hengyang, China). The animals were housed in separate cages in an animal room at 22 sed in separate cages intal Animals at tad libitum. The study was approved by the Ethics Committee of the University of South China (Hengyang, China).
Construction and identification of pQE32/Tp0821. The Tp0821 gene sequences were found in GenBank (https://www. ncbi.nlm.nih.gov/genbank/), and the outer membrane epitopes of the dominant antigen were selected to design primers based on TMPred online programs (http://ch.embnet.org/software/TMPRED_form.html) and ExPasy (http://www.expasy. org/). BamHI and NotI were selected as the restriction sites for the upstream and downstream primers, respectively. The Tp0821 fragment was amplified using PCR. In each case, the PCR mix consisted of dNTPs at $200 \mathrm{M}$ each, $0.25 \mathrm{M}$ each primer, $3 \mathrm{mM} \mathrm{MgCl}_{2}, 10 x P C R$ buffer, $2 \mu \mathrm{l}$ the Tp Nichols strain genomic DNA, 1 U of HotStarTaq DNA polymerase (Qiagen Co., Ltd.) in a total volume of $50 \mu \mathrm{l}$. The following primers: Forward 5'-CGGGATCCTCGTAGGGAAACTCAT T-3' and reverse 5'-CCCAAGCTTGTAGCGTTCCTTCAG AT-3'. Amplification profile was used: $95^{\circ} \mathrm{C}$ denaturation for $50 \mathrm{sec}, 59^{\circ} \mathrm{C}$ annealing for $40 \mathrm{sec}$, and $72^{\circ} \mathrm{C}$ extension for $50 \mathrm{sec}$ for 30 cycles, followed by a 5 min terminal extension at $72^{\circ} \mathrm{C}$. Following enzyme digestion and ligation, pQE32/Tp0821 was constructed. The construct was confirmed by restriction digestion and sequencing.

Expression, purification of rTp0821. The pQE32/Tp0821 was transformed into $E$. coli $\mathrm{M} 15$ with $50 \mathrm{mmol} / 1 \mathrm{CaCl}_{2}$ for $30 \mathrm{~min}$ at $37^{\circ} \mathrm{C}$ and induced by isopropyl $\beta$-d-1-thiogalactopyranoside (IPTG). The denaturation and dialysis of rTp0821 were achieved through ultrasound, inclusion body washing and dissolution with $10 \%$ PBS. The rTp0821 protein was stored at $-70^{\circ} \mathrm{C}$ following detection of its concentration via the Bradford method.

Identification of rTp0821. rTp0821 protein (10 mg) was separated on $12 \%$ SDS-PAGE and transferred onto a nitrocellulose membrane (Bio-Rad Laboratories, Inc., Hercules, CA, USA). The membrane was blocked with $5 \%$ non-fat milk in $10 \mathrm{mM}$ Tris-HCl with $150 \mathrm{mM} \mathrm{NaCl}(\mathrm{pH} \mathrm{8.0)}$ and $0.1 \%$ Tween 20 (TBST) for $2 \mathrm{~h}$ at room temperature. The membrane was then incubated for $1 \mathrm{~h}$ at room temperature with positive serum of second stage syphilis. After three washes with TBST, the membrane was then incubated for $1 \mathrm{~h}$ at room temperature with horseradish peroxidase-conjugated goat anti-human IgG. Following washing 5 times with TBST, protein bands were visualized with ECL prime detection reagent.

Removal and detection of rTp0821 endotoxin. The endotoxin and impurities were removed from rTp0821 through a Detoxi-Gel actifier column. According to the manufacturer's protocol of the chromogenic endpoint Tachypleus Amebocyte Lysate kit, $\mathrm{A}_{545}$ in standard solutions was detected to plot a standard curve, which was confirmed as $|r| \geq 0.098$. The endotoxin content then was computed from the standard curve.

Detection of rTp0821 immunogenicity. Following ultrasonic emulsification with rTp0821 and complete Freund's adjuvant, dorsal subcutaneous multipoint injection was performed in 20 New Zealand rabbits. After 3 weeks, $200 \mu \mathrm{g}$ rTp0821 was booster-immunized with incomplete Freund's adjuvant three times at an interval of 14 days. A negative control group without injection was also included. Prior to each immunization, blood from the ear vein was collected and, 10 days following the 
final immunization, all rabbits were sacrificed by air embolism following anesthesia with $10 \mathrm{mg} / \mathrm{kg}$ ketamine and blood was collected from the heart. The serum was separated by centrifugation at $1,000 \mathrm{x}$ g for $10 \mathrm{~min}$ at $37^{\circ} \mathrm{C}$ and stored at $-20^{\circ} \mathrm{C}$. The experiments were performed using rTp0821-immunized rabbit serum as the primary antibody, HRP-conjugated goat anti-rabbit IgG as the secondary antibody, and normal rabbit serum as the negative control. If the $\mathrm{A}_{450}$ in test group/ $\mathrm{A}_{450}$ in negative control group was $\geq 2.1$, the result was considered positive. The highest fold dilution showing a positive reaction was regarded as the serum titer.

Delayed type hypersensitivity (DTH). rTp0821 (50 $\mu \mathrm{g})$ was injected subcutaneously into left inner thigh of another 20 New Zealand rabbits. The changes at the injected sites were observed. Pyrogen-free water was injected into the corresponding site at the contralateral limb as a control.

Detection of rTp0821 immunoreactivity. rTp0821 (3.0 mg/l) was added to $37^{\circ} \mathrm{C}$ microwell plates, incubated at $4^{\circ} \mathrm{C}$ overnight, and then sealed with $37^{\circ} \mathrm{C}$ phosphate buffer solution containing 5\% skim milk for $2 \mathrm{~h}$, and washed with poly (butylene succinate-co-terephthalate. The 150 samples of normal serum has been diluted 1:100 with PBS containing 1\% BSA, then added bacterial ultrasonic lysis supernatants which were obtained by centrifugation at $14,000 \mathrm{x} \mathrm{g}$ for $20 \mathrm{~min}$ at $4^{\circ} \mathrm{C}$, then were placed at $4^{\circ} \mathrm{C}$ overnight and, following absorption, were added to the microwell plates. Blank wells, positive control wells and negative control wells were also included. Following the addition of HRP-conjugated goat anti-human IgG (diluted 1:20,000) were incubated $30 \mathrm{~min}$ at $37^{\circ} \mathrm{C}$, the detection of $\mathrm{A}_{450}$ of the samples was performed using an enzyme-labeled meter. With the normal serum group as a negative control, the average $\mathrm{A}_{450}+2 \mathrm{~S}$ was used as the positive critical value. Indirect ELISA was used to compare with FTA-ABS to assess the immunoreactivity of the recombinant antigens. $A_{450}$ in test group/ $A_{450}$ in negative control group $\geq 2.1$ was considered a positive result. The highest dilution ratio with a positive reaction was regarded as the titer of these samples.

Statistical analysis. All data are expressed as the mean \pm standard deviation. Independent sample t-tests were used to perform comparisons of pairs within the groups. Multiple groups were compared using a repeated data variance t-test. Statistical analysis was performed using the statistical software program, SPSS, version 13.0 (SPSS, Inc., Chicago, IL, USA). P $<0.05$ was considered to indicate a statistically significant difference.

\section{Results}

Tp0821 gene screening and cloning. Antigenicity is affected by protein polarity, accessibility, approachability, migration, transmembrane region and protein secondary structure. In the present study, predictions based on the TMPred online program and ExPasy showed that the dominant antigen epitopes of the Tp0821 genes were located at the $29-777$ bp base sequence, with a fragment length of $749 \mathrm{bp}$ and encoding 249 amino acids. Using the Tp Nichols standard strain as the template, the Tp0821 genes were cloned into the pQE32 plasmid, and were consistently identified using PCR and sequencing to have correct codon reading frames. These results indicated successful construction of pQE32/Tp0821 (Fig. 1).

Purification, determination of the expression of rTp0821 and removal of endotoxin. The purity and natural conformations of recombinant proteins determine whether the body can elicit an immune response. As shown in Fig. 2A, following induced expression at $37^{\circ} \mathrm{C}$ with $1.0 \mathrm{mM}$ IPTG, the rTp0821 recombinant proteins were present in the ultrasonic precipitation, but not in the supernatants. The inclusion body was separated, preliminarily purified and, following denatured dissolution, was eluted on an Ni-NTA column. A $29 \mathrm{kDa}$ protein with a similar size to that of the predesigned molecular weight was identified (Fig. 2B). The rTp0821 concentration was measured using a BCA protein assay as $470 \mu \mathrm{g} / \mathrm{ml}$. Using the rTp0821 immune serum as a primary antibody, a target protein with size of $\sim 29 \mathrm{kDa}$ was identified using western blot analysis (Fig. 2C). These results indicated that rTp0821 was effectively expressed in E. coli BL21. An endotoxin removing gel was used to remove endotoxin from the purified rTp0821, and the endotoxin content was measured using a Tachypleus Amebocyte Lysate kit as <0.04 EU/ml.

Immunogenicity and DTH of rTp0821. Following the initial immunization of rabbits with rTp0821, no rTp0821-specific antibody was found in the serum using ELISA. Following the second immunization, the titer of rTp0821 antibody increased marginally, with a titer of 1:400. Following the booster immunization, the antibody titer increased to $1: 6,400$, and the serum positive reaction rate was $8 / 8$. The negative control group was immunized four times with Freund's adjuvant, and no rTp0821-specific antibody was detected in the serum (Fig. 3). These results indicated that rTp0821 had high immunogenicity.

At 3 days following injection with the recombinant proteins, the rabbits began to show DTH, and the injected sites were swollen by $\sim 1.5 \times 1.5 \mathrm{~cm}$. The skin at the swollen sites felt warmer to touch, compared with the corresponding contralateral sites. The swelling disappeared at 6-10 days. No DTH occurred at the control sites treated with normal saline.

Immunoreactivity of rTp0821. The immunoreactivity of rTp0821 was analyzed using western blot analysis, with positive serum from patients with second-stage syphilis as the primary antibody. The induced product and the purified protein showed a single specific band at the same site, which is not observed on the blot with the non-induced product (Fig. 4).

An indirect ELISA method, using rTp0821 recombinant proteins as the envelope antigen, was used to detect Tp IgG antibody. This method was used for random detection in the serum of 150 healthy subjects, and the average $\mathrm{A}_{450}$ was 0.203 (SD, 0.033), with a positive critical value (average $\left.\mathrm{A}_{450}+2 \mathrm{xSD}=0.203+0.066=0.269\right)$. The 150 negative serum samples and 150 positive serum samples were also detected following FTA-ABS. As shown in Table I, ELISA, compared with FTA-ABS had a sensitivity of $92.6 \%(139 / 150)$ and specificity of $98.6 \%(148 / 150)$, with a coincidence rate of $95.6 \%$. Therefore, the indirect ELISA had a significantly lower rate of detection, compared with FTA-ABS. These results indicated that rTp0821 had a high level of antigenicity. 

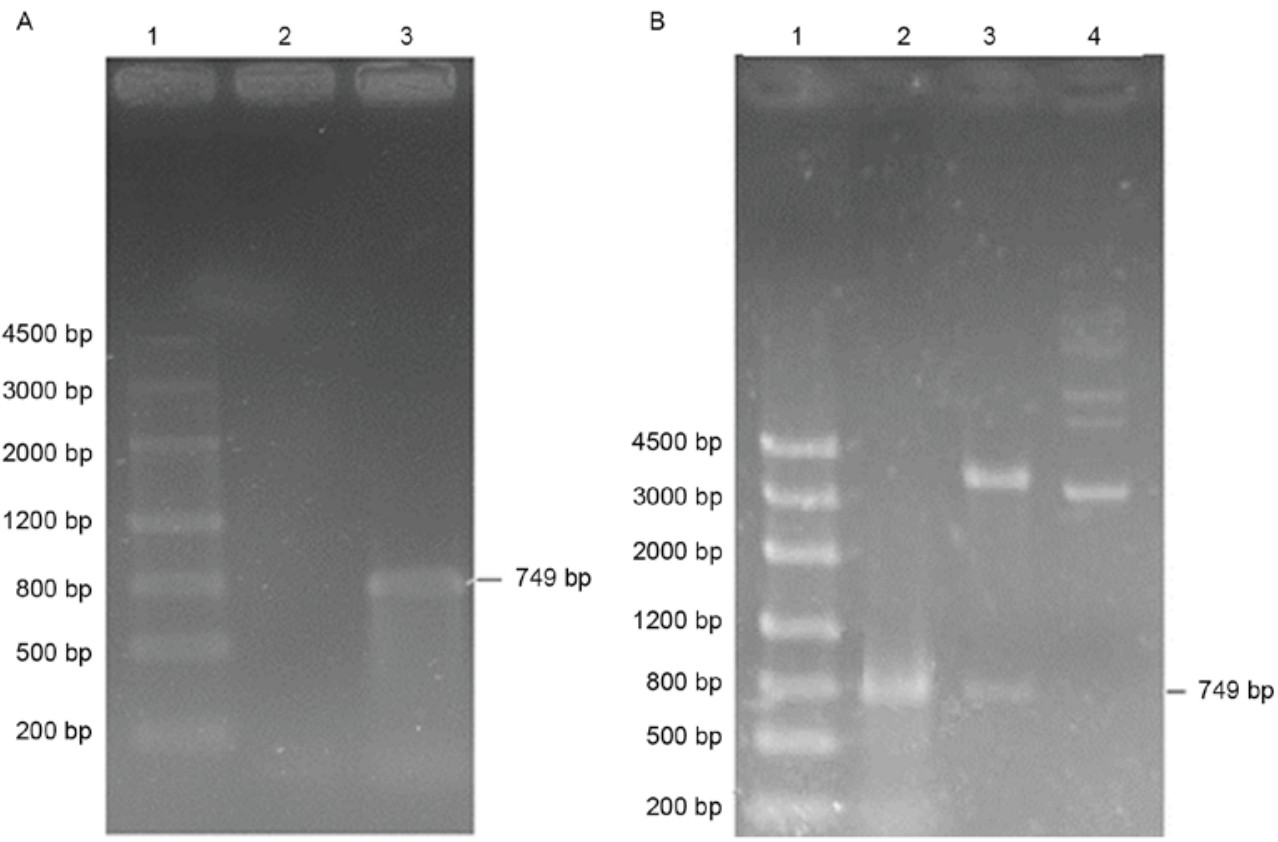

Figure 1. Construction and identification of Tp0821. (A) Electrophoretic analysis of the Tp0821 PCR product on 1\% agarose gel. Band 1, protein marker; band 2, negative control; band 3, PCR product. (B) Restriction mapping of recombinant plasmid pQE32/Tp0821 digested with BamHI and HindIII. Band 1, DNA marker; band 2, PCR product; band 3, recombinant plasmid pQE32/Tp0821 digested with BamHI and HindIII; band 4, recombinant plasmid pQE32/Tp0821. PCR, polymerase chain reaction.

A

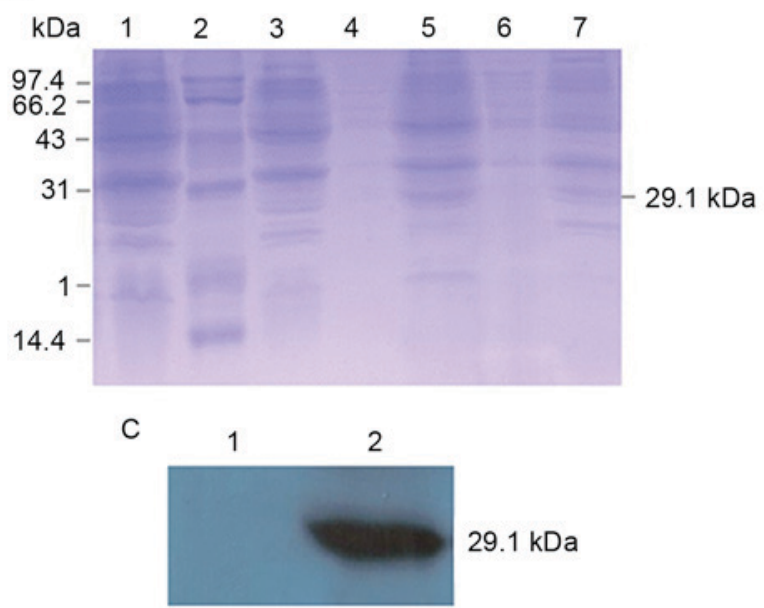

B

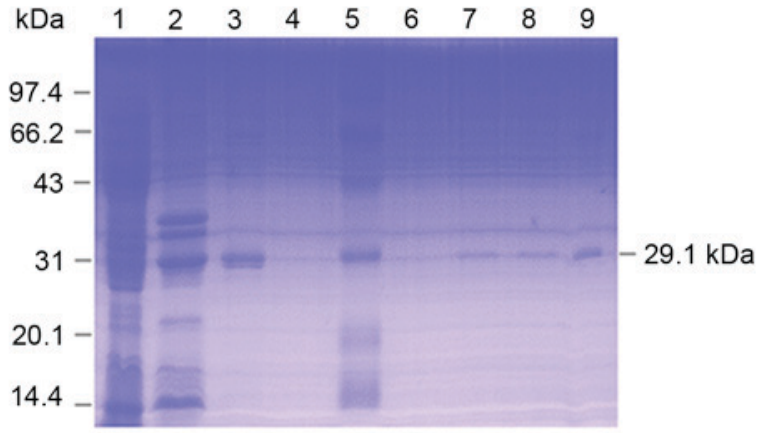

Figure 2. Expression, purification and identification of rTp0821. (A) SDS-PAGE of expressed pQE32/Tp0821 in E. coli M15. 1, non-induced bacteria with $\mathrm{pQE} 32 / \mathrm{Tp} 0821$ at $37^{\circ} \mathrm{C} ; 2$, protein marker; 3, non-induced bacteria with pQE32/Tp0821 at $27^{\circ} \mathrm{C} ; 4$, supernatant of broken induced bacteria with pQE32/Tp0821 at $37^{\circ} \mathrm{C} ; 5$, precipitation of broken induced bacteria with $\mathrm{pQE} 32 / \mathrm{Tp} 0821$ at $37^{\circ} \mathrm{C} ; 6$, supernatant of broken induced bacteria with $\mathrm{pQE} 32 / \mathrm{Tp} 0821$ at $27^{\circ} \mathrm{C}$; 7, precipitation of broken induced bacteria with pQE32/Tp0821 at $27^{\circ} \mathrm{C}$. (B) Purification of Tp0821 recombinant protein under denaturing conditions. 1 , supernatant of broken Tp0821 recombinant protein; 2, precipitation of broken Tp0821 recombinant protein; 3, cell lysate flow-through; 4, protein marker; 5, buffer $\mathrm{C}$ washes; 6-7, buffer D washes; 8-9, buffer E eluates. (C) Western blot analysis of recombinant protein. 1, non-induced recombinant bacteria with pQE32/Tp0821; 2, induced bacteria with pQE32/Tp0821.

\section{Discussion}

The in vitro cultivation of $\mathrm{Tp}$ has not been possible, as the limited antigen source has restricted investigations on the diagnosis and pathogenic mechanism of Tp (17). As reported, the outer membrane protein (OMP) and lipoprotein, as the primary immune components of $\mathrm{Tp}$, can induce a marked immune response in the body and are important in the pathopoiesis and infection of $\mathrm{Tp}$ (18-20). In the present study, based on literature and application software, the dominant epitope fragment of Tp OMP, Tp0821, was screened out, which had high antigenicity. Nucleotide sequences of Tp0821did not show any homology with other bacteria by the Basic Local Alignment Search Tool (https://blast.ncbi.nlm.nih.gov/Blast.cgi), therefore, it was possible for use as an antigen candidate molecule for the detection of syphilis antibody.

The selection of expression vectors is vital in the expression and purification of recombinant proteins. In the present 
Table I. Comparison of 300 serum samples analyzed using indirect ELISA and FTA-ABS.

\begin{tabular}{lccc}
\hline & \multicolumn{3}{c}{ Indirect ELISA } \\
\cline { 2 - 4 } FTA-ABS & Positive & Negative & Total \\
\hline Positive & 139 & 11 & 150 \\
Negative & 2 & 148 & 150 \\
Total & 141 & 159 & 300 \\
\hline
\end{tabular}

Data obtained from the number of surviving rabbits in each group within $72 \mathrm{~h}$. Data are presented as the mean \pm standard deviation from three independent experiments. The two methods were compared using paired $\chi^{2}$ four-fold table, $\chi^{2}=4.92$; coincidence rate $(139+148) /(141+159)=95.6 \%$. ELISA, enzyme-linked immunosorbent assay; FTA-ABS, fluorescent treponemal antibody-absorption.

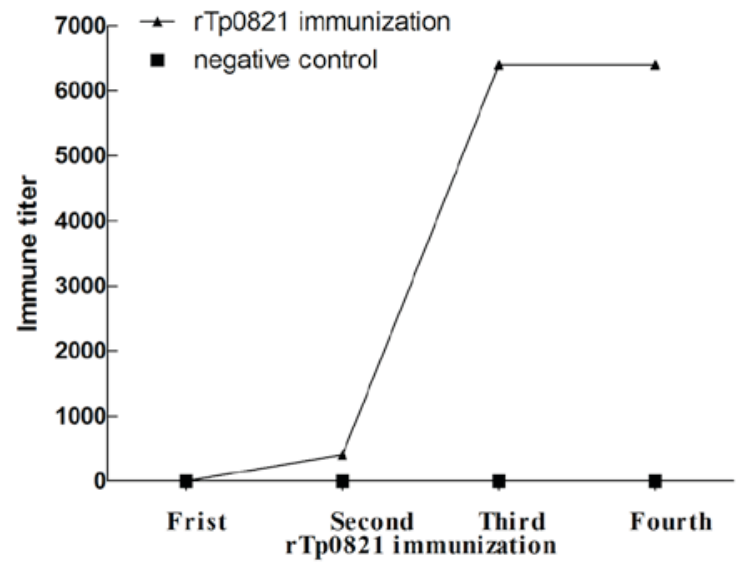

Figure 3. Immune titer of rTp0821 antibody. $\mathrm{A}_{450}$ in test group $/ \mathrm{A}_{450}$ in negative control $\geq 2.1$ indicated a positive result. The highest fold dilution showing a positive reaction was regarded as the serum titer compared to controls.

study pQE32, from the pDS plasmid family, was selected, which contains a 6-histidine coding sequence and can express exogenous recombinant proteins with a 6-histidine purification tag. pQE32 has minimal effect on protein synthesis, secretion, space conformation and immunogenicity (21), nor does it affect the structure and function of recombinant proteins, however, it enables high-purity recombinant proteins to be obtained.

In the present study, purified rTp0821-immune New Zealand rabbits were used for the acquisition of immune polyvalent serum, which was detected with a high specific polyclonal titer using indirect ELISA. These results indicated that the recombinant proteins had high immunogenicity and led to the New Zealand rabbits producing a marked humoral immune response. The immunization effect was gradually enhanced with the increased number of immunizations. The results of the western blot analysis revealed that the purified recombinant proteins reacted with the Tp-positive infected serum, confirming its high immunoreactivity. Using enveloping enzyme-linked plates with rTp0821, an indirect ELISA method was used for the detection of $\mathrm{Tp}$ IgG, and identified a positive critical value for comparison with FTA-ABS. Despite a lower detection rate, compared with FTA-ABS, indirect
1

2

3

$29.1 \mathrm{kDa}$

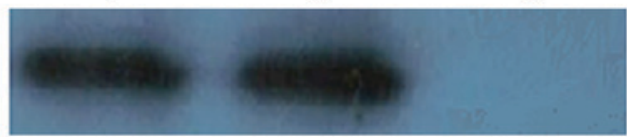

Figure 4. Western blot analysis of Tp0821 recombinant protein. 1, purified protein; 2 , induced recombinant bacteria with $\mathrm{pQE} 32 / \mathrm{Tp} 0821 ; 3$, non-induced bacteria with pQE32/Tp0821.

ELISA had a high coincidence rate, indicating its potential in the detection of syphilis in serum.

At present, the mechanism underlying anti-Tp infection in the body remains to be fully elucidated. As described above, the primary immune protection mechanism of syphilis is DTH and during the reaction against $\mathrm{Tp}$, cell immunity is more important than humoral immunity, particularly $\mathrm{T}$ cells (22). The DTH of rTp0821 recombinant proteins qA positive, indicating that rTp0821 induced a specific cell immune response and may be selected as a Tp vaccine candidate protein.

The present study provided preliminarily evidence that rTp0821 recombinant proteins had high immune reactivity. The ELISA method, based on rTp0821 recombinant proteins, showed high sensitivity and specificity, and may be used in clinical and epidemiological investigations. However, its sensitivity and specificity requires validation through the detection of abundant clinical serum samples in addition to improvements in the method. The present findings are somewhat inconsistent, which may be attributed to three possible factors: i) Following treatment with antibiotics, the antibody titer is significantly reduced within a period of time, which reduces the antibody detection rate; ii) the detection method is based on a single epitope antigen, which leads to limited coverage of the antigen epitope, reducing detective sensitivity and specificity; iii) the critical value may be too high $(23,24)$. If the critical value can be appropriately reduced without altering the specificity, it is possible to improve the sensitivity. Future investigations aim to perform chimeric recombination of Tp0821 with dominant antigen epitopes of other membrane protein molecules, for example, TpN47, TpN17, TpN15 and TmpA, and to investigate the roles of multi-epitope chimeric recombinant proteins in the serologic diagnosis of syphilis $(25,26)$. This is likely to lead to further improvements in the sensitivity and specificity of the method, and establish a rapid, simple, sensitive, specific and stable early-stage Tp diagnosis kit.

The mechanism underlying anti-Tp infection in the body remains to be fully elucidated, and residual endotoxins during protein expression and purification may interfere with experiments. Preliminary experiments showed that an endotoxin concentration threshold in vivo $>0.005 \mathrm{ng} / \mathrm{ml}$ can induce endogenous pyrogen. Therefore, to detect the toxic effects of target proteins on cells, cellular interference from endotoxins requires elimination. In the present study, Detoxi-Gel ${ }^{\mathrm{TM}}$ endotoxin-removing gel was used to remove the majority of endotoxins remaining in the purified proteins.

Membrane lipoprotein is an important component in $\mathrm{Tp}$ pathopoiesis (11). It has also been found that, in infected tissues, lymphocytes and particularly macrophages showed marked phagocytosis of $\mathrm{Tp}$ (10). The Tp membrane lipoprotein is considered an important component of Tp pathopoiesis due 
to three possible pathogenic mechanisms: i) It can activate mononuclear cells/macrophages and endothelial cells, and exhibit potent proinflammatory activity; ii) it promotes $\mathrm{T}$ cell proliferation via the Toll-like receptor-2-based activation of immune effector cells; iii) it promotes the adhesion and invasion of Tp on host cells, and may be involved in immune evasion $(11,27)$.

\section{Acknowledgements}

This study was supported by the National Natural Science Foundation of China (grant no. 31100137), the Natural Science Foundation of Hunan Province (grant no. 14JJ7044), the Project Foundation of Health Department of Hunan Province (grant nos. B2011-130 and B2013-133), the 12th Five-Year Technology Innovation Team in University of South China, Project from Changzhou Health Bureau (grant no. WZ201125). The authors would like to thank Dr Xiaoxing You (Pathogenic Biology Institute, University of South China, Hengyang, China) for their technical assistance and advice.

\section{References}

1. Lewis DA and Lukehart SA: Antimicrobial resistance in Neisseria gonorrhoeae and Treponema pallidum: Evolution, therapeutic challenges and the need to strengthen global surveillance. Sex Transm Infect 87 (Suppl 2): ii39-ii43, 2011.

2. Stamm LV: Yaws: 110 years after Castellani's discovery of Treponema pallidum subspecies pertenue. Am J Trop Med Hyg 93: 4-6, 2015.

3. Dai T, Li K, Lu H, Gu X, Wang Q and Zhou P: Molecular typing of Treponema pallidum: A 5-year surveillance in Shanghai, China. J Clin Microbiol 50: 3674-3677, 2012.

4. Smajs D, Norris SJ and Weinstock GM: Genetic diversity in Treponema pallidum: Implications for pathogenesis, evolution and molecular diagnostics of syphilis and yaws. Infect Genet Evol 12: 191-202, 2012.

5. Tipple C, Jones R, McClure M and Taylor G: Rapid Treponema pallidum clearance from blood and ulcer samples following single dose benzathine penicillin treatment of early syphilis. PLoS Negl Trop Dis 9: e0003492, 2015.

6. Shields M, Guy RJ, Jeoffreys NJ, Finlayson RJ and Donovan B: A longitudinal evaluation of Treponema pallidum PCR testing in early syphilis. BMC Infect Dis 12: 353, 2012.

7. Busse C, Navid MH, Strubel A and Schnitzler P: Evaluation of a new recombinant antigen-based Virotech Treponema pallidum screen ELISA for diagnosis of syphilis. Clin Lab 59: 523-529, 2013.

8. Knauf S, Dahlmann F, Batamuzi EK, Frischmann S and Liu H: Validation of serological tests for the detection of antibodies against Treponema pallidum in nonhuman primates. PLoS Negl Trop Dis 9: e0003637, 2015.

9. Liu C, Ou Q, Chen H, Chen J, Lin S, Jiang L and Yang B: The diagnostic value and performance evaluation of five serological tests for the detection of Treponema pallidum. J Clin Lab Anal 28: 204-209, 2014

10. Zhang RL, Wang QQ, Zhang JP and Yang LJ: Tp17 membrane protein of Treponema pallidum activates endothelial cells in vitro. Int Immunopharmacol 25: 538-544, 2015.

11. Brautigam CA, Deka RK, Liu WZ and Norgard MV: Insights into the potential function and membrane organization of the TP0435 (Tp17) lipoprotein from Treponema pallidum derived from structural and biophysical analyses. Protein Sci 24: 11-19, 2015.

12. Lin LR, Fu ZG, Dan B, Jing GJ, Tong ML, Chen DT, Yu Y, Zhang CG, Yang TC and Zhang ZY: Development of a colloidal gold-immunochromatography assay to detect immunoglobulin $\mathrm{G}$ antibodies to Treponema pallidum with TPN17 and TPN47. Diagn Microbiol Infect Dis 68: 193-200, 2010.
13. Peng RR, Yin YP, Wei WH, Wang HC, Zhu BY, Liu QZ, Zheng HP, Zhang JP, Huang SJ and Chen XS: Molecular typing of Treponema pallidum causing early syphilis in China: A cross-sectional study. Sex Transm Dis 39: 42-45, 2012.

14. Aktas G, Young H, Moyes A and Badur S: Evaluation of the fluorescent treponemal antibody absorption test for detection of antibodies (immunoglobulins $\mathrm{G}$ and $\mathrm{M}$ ) to Treponema pallidum in serologic diagnosis of syphilis. Int J STD AIDS 18: 255-260, 2007.

15. Brinkman MB, McKevitt M, McLoughlin M, Perez C, Howell J, Weinstock GM, Norris SJ and Palzkill T: Reactivity of antibodies from syphilis patients to a protein array representing the Treponema pallidum proteome. J Clin Microbiol 44: 888-891, 2006.

16. Deka RK, Neil L, Hagman KE, Machius M, Tomchick DR, Brautigam CA and Norgard MV: Structural evidence that the 32-kilodalton lipoprotein ( $\mathrm{Tp} 32$ ) of Treponema pallidum is an L-methionine-binding protein. J Biol Chem 279: 55644-55650, 2004.

17. Salado-Rasmussen K, Cowan S, Gerstoft J, Larsen HK, Hoffmann S, Knudsen TB, Katzenstein TL and Jensen JS: Molecular Typing of Treponema pallidum in Denmark: A Nationwide Study of Syphilis. Acta Derm Venereol 96: 202-206, 2016.

18. Naqvi AA, Shahbaaz M, Ahmad F and Hassan MI: Identification of functional candidates amongst hypothetical proteins of Treponema pallidum ssp. pallidum. PLoS One 10: e0124177, 2015.

19. Zhang RL, Zhang JP and Wang QQ: Recombinant Treponema pallidum protein Tp0965 activates endothelial cells and increases the permeability of endothelial cell monolayer. PLoS One 9: e115134, 2014.

20. Stamm LV and Drapp RL: A synthetic lymph node containing inactivated Treponema pallidum cells elicits strong, antigen-specific humoral and cellular immune responses in mice. Pathog Dis 70: 88-94, 2014.

21. Liu WX, Hu S, Qiao ZJ, Chen WY, Liu LT, Wang FK, Hua RH, $\mathrm{Bu} \mathrm{ZG}$ and Li XR: Expression, purification, and improved antigenic specificity of a truncated recombinant bp26 protein of Brucella melitensis M5-90: A potential antigen for differential serodiagnosis of brucellosis in sheep and goats. Biotechnol Appl Biochem 58: 32-38, 2011.

22. Carlson JA, Dabiri G, Cribier B and Sell S: The immunopathobiology of syphilis: The manifestations and course of syphilis are determined by the level of delayed-type hypersensitivity. Am J Dermatopathol 33: 433-460, 2011.

23. Sun A, Fan X, Shen X, Tang R and Yan J: Recombinant expression of the fusion antigen based on Treponema pallidum TpN17 and TpN47 epitope peptides and establishment and application of the associated ELISA. Sheng Wu Gong Cheng Xue Bao 25: 1187-1194, 2009 (In Chinese).

24. Sun AH, Fan XL, Mao YF, Peng MF, Fan CH and Yan J: Comparison of serological detection effects of ELISA using rTpN17 or rTpN47 of Treponema pallidum as antigen with that of TPHA and TRUST. Zhejiang Da Xue Xue Bao Yi Xue Ban 37: 67-72, 2008 (In Chinese).

25. Sun AH, Mao YF, Hu Y, Sun Q and Yan J: Sensitive and specific ELISA coated by TpN15-TpN17-TpN47 fusion protein for detection of antibodies to Treponema pallidum. Clin Chem Lab Med 47: 321-326, 2009.

26. McGill MA, Edmondson DG, Carroll JA, Cook RG, Orkiszewski RS and Norris SJ: Characterization and serologic analysis of the Treponema pallidum proteome. Infect Immun 78: 2631-2643, 2010

27. Luthra A, Zhu G, Desrosiers DC, Eggers CH, Mulay V, Anand A, McArthur FA, Romano FB, Caimano MJ, Heuck AP, et al: The transition from closed to open conformation of Treponema pallidum outer membrane-associated lipoprotein TP0453 involves membrane sensing and integration by two amphipathic helices. J Biol Chem 286: 41656-41668, 2011. 